\title{
Cognitive Scales and Mental Models for Inclusive Design
}

\author{
Umesh Persad $^{1}$, Patrick Langdon ${ }^{1}$, P John Clarkson ${ }^{1}$, David Brown ${ }^{2}$ \\ ${ }^{1}$ Cambridge Engineering Design Centre, Department of Engineering, Cambridge \\ University, Trumpington Street, Cambridge, CB2 1PZ, United Kingdom \\ \{up209@eng.cam.ac.uk,pml24@eng.cam.ac.uk, pjc10@eng.cam.ac.uk \} \\ ${ }^{2} \mathrm{AI}$ in Design Group, Computer Science Dept., Worcester Polytechnic Institute, 100 \\ Institute Rd., Worcester, MA 01609, USA \\ $\{$ dcb@cs.wpi.edu
}

\begin{abstract}
In keeping with a user capability and product demand approach to product assessment, this paper examines the cognitive demands placed on users when interacting with consumer products. The eventual aim is to develop a set of cognitive capability scales that could be used in the analytical evaluation of product interfaces. We explore the dimensions of cognitive capability relevant to product interaction and describe how these may be used to evaluate a given design. ??Further work addresses quantitative measurement of cognitive capabilities and predictive validation of capability scales.
\end{abstract}

Keywords: Inclusive Design, Product Evaluation, Cognitive Assessment, working memory

\section{Introduction}

Inclusive design is defined as the "design of mainstream products and/or services that are accessible to, and usable by, people with the widest range of abilities within the widest range of situations without the need for special adaptation or design" [1]. It is therefore a design philosophy that aims to consider the needs and capabilities of older and disabled users in the design process. In addition, the approach is focused on mainstream product design as apposed to assistive technology by avoiding aids, adaptations and stigmatising designs [2]. The concept of inclusive design is similar to Universal Design which is popular in the United States and Japan. As the term 'universal' may connote a 'one size fits all' approach, inclusive design attempts to include users with reduced functional capability in mainstream product design without sacrificing product aesthetics and desirability. Recognising that a completely inclusive product is an ideal as opposed to a practically achievable result, the focus of inclusive design is on implementing a user-centred design process. The result of such a process should be improved product designs that minimise the exclusion of less capable populations [3]. 
Designers require information on the range of human sensory, cognitive and motor capabilities in order to evaluate their designs [4]. Traditionally, human factors information is delivered in the form of guidelines and handbooks [5-7]. However, recent research has shown that designers require quantitative data on the numbers of people with functional capability loss presented in accessible and visual formats [8, 9]. Currently, there is a lack of a complete, unified source of user capability data that could be used to evaluate design concepts. The following section gives the background to this research and demonstrates the need for the development of cognitive capability scales.

\section{Background}

The ideas of user capability and product demand provide a useful framework for design evaluation. Figure 1 shows this framework where the sensory, cognitive and motor demands made by a product are compared to the capability levels of the target user population $[4,10]$. In order to measure the level of compatibility between the user and the product, various evaluation methods can be employed. These methods can be roughly classified into empirical methods and analytical methods [11].

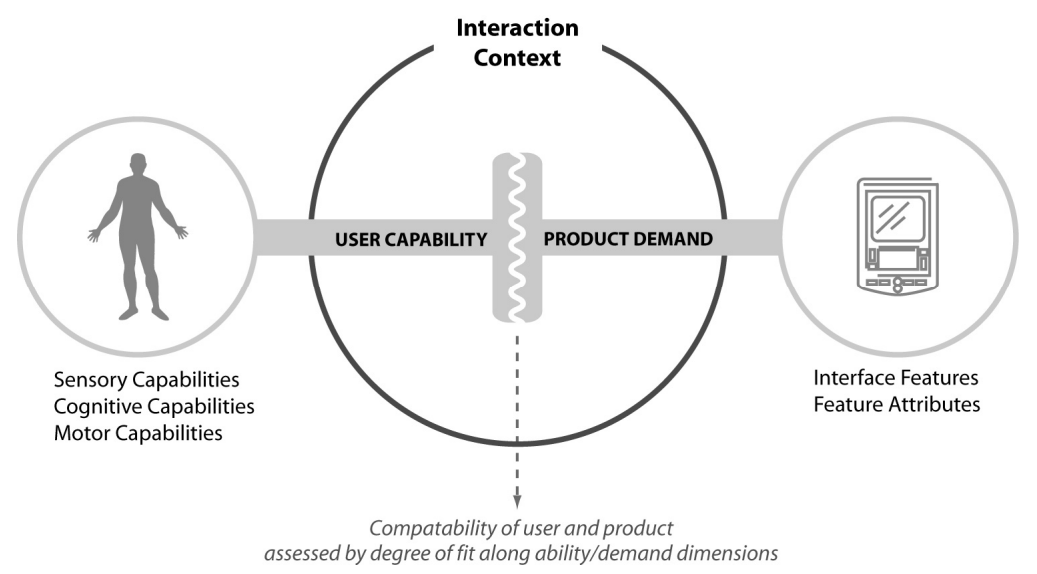

Fig. 1. A model for evaluation based on user capability and product demand.

Empirical evaluation methods measure design performance in actual usage scenarios by having users perform tasks with a product design. Various performance metrics are recorded such as time taken, number and type of errors, and subjective impressions. Analytical methods rely on the detailed inspection of the product and intended scenarios of use without direct user involvement. Analytical methods can range from applying a relatively cheap and quick heuristic evaluation to the use of predictive engineering models of user behaviour. Ideally there should be a balance of both analytical and empirical evaluation methods in the design process depending on the resources available. Though the importance of user involvement in the design process 
cannot be overemphasised, there is value in utilising analytical methods in the evaluation process due to constraints of time, cost and logistical difficulties in recruiting and testing with real users [12]. In addition, analytical methods are especially advantageous in the inclusive design process where a group and population view on user capability is required $[13,14]$. We therefore concentrate on the development of methods to analytically evaluate consumer products for accessibility and usability.

Keates [2] describes human functional capability in the three psychological dimensions of sensory, cognitive and motor capability based on the Model Human Processor [15]. This provides a useful basis for engineering model of capability for product evaluation, even though the three dimensions are not independent and do interact in the performance of real world tasks. A unique source of capability data exists in the global functional capability scales developed for the 1996/97 Great Britain Disability Follow-up Survey (DFS) [16]. Though the scales lack the granularity and completeness to evaluate all aspects of consumer products, they provide a unique set of multivariate capability data that is representative of the Great Britain population. Langdon et al. [17] describe an attempt to derive improved capability scales for assessing product interaction based on current approaches in cognitive psychology. It was shown that this was possible within the confines of the variables measured in the survey and further work is required to develop a more complete set of design relevant cognitive capability scales.

In this paper, we focus on the cognitive demands of consumer products via an analysis of product features and user tasks. We outline various categories of cognitive demand that are relevant to product interaction. In turn, these form scales of user cognitive capability that should be of adequate scope and comprehensiveness to evaluate a large range of consumer products. They should allow for valid predictions of user exclusion and difficulty to be made under normal assumptions of use. The predictions should also be sensitive to changes in product attributes allowing a designer to make changes to the design and see the effects on the predictions. Finally, the scales should also be usable by designers.

\section{Cognitive Capabilities}

Carroll [18] defines capability as follows: "As used to describe an attribute of individuals, ability refers to the possible variations over individuals in the liminal levels of task difficulty (or in derived measurements based on such liminal levels) at which, on any given occasion in which conditions appear favourable, individuals perform successfully on a defined class of tasks." This definition implies that cognitive capability is measured by the threshold levels of performance only with reference to a specified class of tasks. For our purposes, these tasks involve the mental processes that are required in product interaction. This involves retrieving previous knowledge of a particular product's features and how the product works, and using this knowledge together with task demands and product perception to form mental models in working memory [19]. When a user interacts with a product, there is 
a cyclic process of action, execution, and perception of the effects of the action and evaluation of the effects in terms of the user's goals [20,21].

Various cognitive architectures have been proposed that attempt to describe the information processing sub-systems involved in cognition [22-25]. Based on these architectures, a simplified model of cognition was derived as shown in Figure 2. It consists of a Working Memory of modal stores and a central executive. The central executive controls attentional resources and facilitates the storage and retrieval of information in Long Term Memory. Using this model, measures of cognitive demand in the domains of Working Memory and Attention, Long-Term Memory and Knowledge and Language and Communication are proposed for assessing the cognitive demand of products.

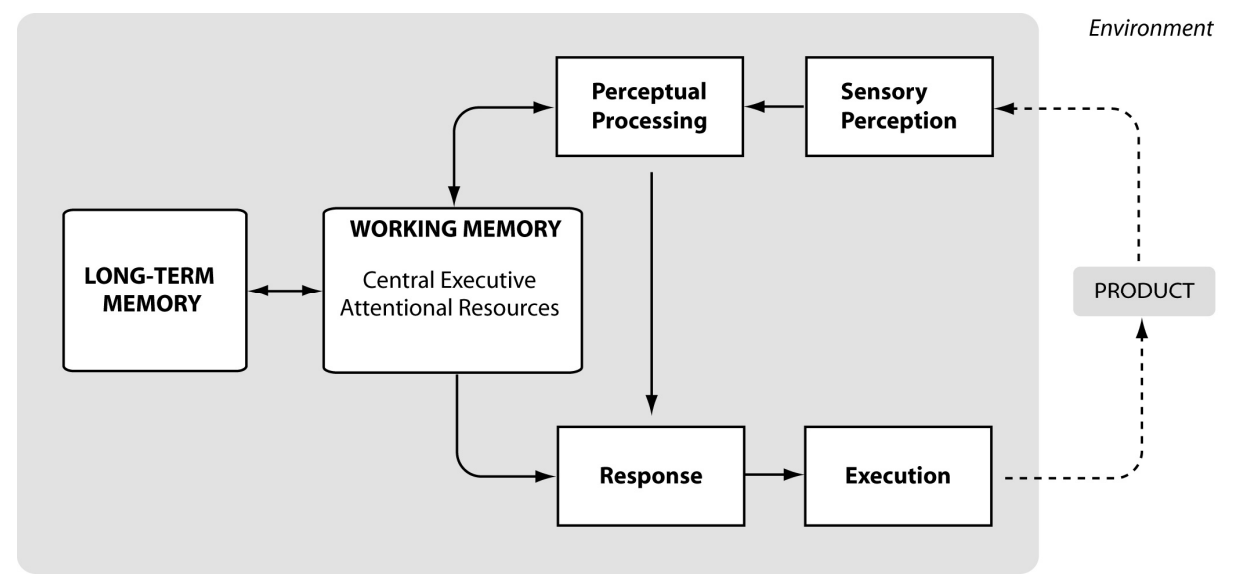

Fig. 2. A simple model of cognition incorporating sensation, perception, working and long term memories and response selection and execution.

\subsection{Working Memory and Attention}

Working memory can be considered as a scratch-pad or rough working area for items being attended to [26]. It is organised by different modalities of storage such as visual-spatial and auditory-verbal. Working Memory has been found to have limited capacity for stored information and a duration of between 10-15 seconds. The general capacity of Working Memory system has been estimated to be around five to nine chunks of information [26]. However, more complex items such as sentences, procedures, or images can be remembered as if they were individual elements when chunked. This is after prolonged use has caused them to become well established in Long Term Memory.

Another important characteristic of the Working Memory model is that the central executive is assumed to have limited resources of attention. This can be overloaded by either increasing the volume of individual items to deal with or the number of simultaneous activities that require attention. Therefore Working Memory and 
attention capacities are the limiting factors when interacting with products. Thus two important performance measures for the working memory system are storage capacity in terms of the number of chunks that can be held and speed and accuracy of processing.

\subsection{Long Term Memory}

Long term memory is a permanent store for knowledge gathered from experience. The type of knowledge stored can be classified into various types including semantic memory, episodic memory and procedural memory. It is useful to distinguish between knowledge of product features and how the product works, versus knowledge on how to use the product in terms of action sequences that will achieve goals. These two types of knowledge are inter-related, and are both used when interacting with products. Therefore, recognition and recall capabilities are the limiting factors in the performance of Long Term Memory. Measures of recognition capability are required when comparing visible product features to information stored in Long Term Memory. Recall capability measures are needed for determining users' ability to retrieve stored knowledge about product features and behaviour.

\subsection{Interaction and Mental models}

Working Memory and Long-Term Memory systems work together when faced with an episode of interaction. Users are assumed to construct mental models in working memory based on previous knowledge cued by current environmental characteristics $[19,27]$, and use this representation as they proceed through the interaction. These models may reflect their understanding of the behaviour of the product and how it is to be used $[19,28]$.

The concept of mental models has received significant attention in the HCI and applied cognitive science literature. It has been found that mental models can be incomplete, unstable, often unscientific and parsimonious and vary in complexity depending on the degree of previous experience [28]. Because of this dependence on previous experience and continuous modification through successive interactions, mental models can be difficult to capture [19].

From a practical standpoint, we use the concept of a mental model as a device that could enable the operational estimation of cognitive information processing demands. We thus use the mental model concept in a representation that captures the demanded mental model of device usage. We posit cognitive processes associated with interaction that act on this representation during the guidance of action. The mental model therefore should consist of a representation of: (1) knowledge of the various interface features and how they work and (2) a representation of the action sequences necessary for moving from an initial state to a goal state. Because Working Memory is limited, there will be a limit to the complexity of the mental model in mind and the mental operations that can be performed on it at any one time. A representation of the demanded mental model could also be used to analyse and identify states in the interaction that could mentally overload the user. 


\subsection{Language and Communication}

Language and communication capabilities involve the comprehension and expression of verbal and written language. An assessment of language comprehension capability is necessary when reading labels and product manuals. It is also employed to interpret verbal messages from a product or system. An assessment of language communication capabilities is important for giving spoken commands to a product. For product evaluation, the primary concern is with linguistic communication in speech and sentence construction as these are most commonly employed in product design.

\section{Case Study}

We now describe a case study involving the analysis of a simple toaster for cognitive demands. Toasters are common devices found in many homes consisting of a relatively straightforward interface. A simple toaster is shown in Fig 3 consisting of a chassis, slots, slider, rotary heat control and a stop button.
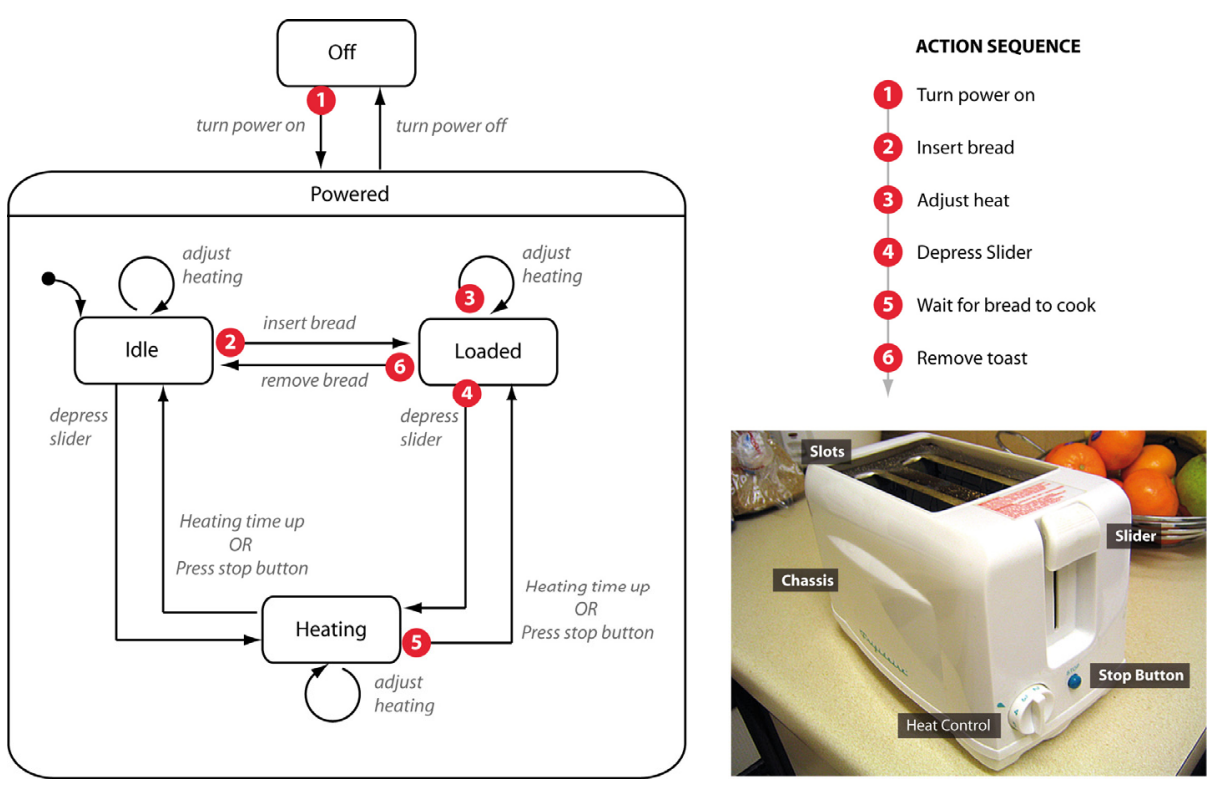

Fig. 3. Diagram showing a state chart of toaster reactive behaviour (left) with a demanded action sequence overlaid, the demanded action sequence (top right), and a picture of the toaster with relevant interface features labelled (bottom right)

The figure also shows a representation for the reactive behaviour of the toaster using a statechart. Statecharts are a part of the Unified Modelling Language and thus provide a standardised way of representing the response of the toaster to various user actions. 
Statecharts are also commonly used to specify the design of reactive systems and are familiar to designers of embedded systems. The demanded user action sequence for making toast is also shown in Fig 3 consisting of six steps. An objected oriented representation of the interface features and associated properties could also be developed for the toaster using UML notation. However, for the purposes of this paper, the statechart alone is shown.

The representation can be used to derive the action sequences that form users' mental models by analysing variations to the demanded action sequence. For example, if step 1 of powering the toaster is omitted, an error of omission occurs and the toaster slider will not activate. In addition, the state based representation of the use process allows for the evaluation of adequate feedback on each state of the device. By examining the toaster, it is evident that inadequate feedback is provided in the powered state and in the heating state. An improvement to the design is immediately possible by including a visual signal such as an indicator light to indicate that the toaster is powered. An auditory feedback on reaching the bread cooked state would be another improvement to indicate to the user that the toast is finished, which can also benefit visually impaired users.

\subsection{Working memory and attention demands}

The action sequence shown in Fig 3 represents first time use. If the assumption is made that people commonly leave their toaster on a particular heat setting most of the time and the power is on; the user has to plan for four steps: (1) put bread in, (2) depress slider, (3) wait for bread to cook and (4) remove toast. Thus the user is only required to keep track of the state of the bread. The attention demands are therefore relatively low assuming that there are no other distractions in the cooking environment that would be outside the control of the toaster designer. However, apart from the toast popping up, the toaster does not signal that the bread has been toasted. As previously mentioned, an auditory alert could be a design improvement, especially if users may attend to other tasks while bread is toasting.

The toaster also does not demand a high working memory capacity. The number of steps in the action sequence can be used as an indicator of the level of demand on planning capabilities of the user as he/she plans through the sequence of actions that will be performed on the toaster. There are also no time demands for task actions that could exceed the Working Memory time limit on storage. For any given design, the aim should be to reduce the number of actions that users have to perform in order to reach their goals thus reducing the cognitive demands of planning and Working Memory.

\subsection{Long Term memory Demands}

Toasters are relatively common consumer products, and the design of the example toaster follows the traditional toaster form factor with slots at the top and a slider at the side (Fig. 3). The interfaces features of slots, slider, rotary control and button are standard features that are also straightforward and prevalent on other toaster designs. 
Thus the demands of the toaster on the knowledge and Long Term Memory of users is assumed to be relatively low. However, the toaster introduces a prospective memory demand where the user is required to remember to remove the toast once it is finished cooking. Again, the simple toaster does not signal that the bread has been toasted, or is being toasted, apart from the toast popping up. A more distinct visual or auditory alert could be a design improvement.

\subsection{Communication and Language Demands}

Communication and language demands are relatively low for the toaster because no graphical symbols are used. The user is only required to read and understand the 'STOP' button label and the red text of a safety sticker on the toaster. Reading the safety sticker is not essential to using the toaster, so within the defined task bounds the toaster does not demand a high degree of written comprehension capability. Visuospatial communications demands are also relatively low for the toaster as no graphical symbols are used.

\subsection{Learning and Feedback}

Feedback plays an important role in the learnability of a product. As previously mentioned, the representation of mental models allows for the evaluation of adequate feedback on each state of the device. This supports the user forming an adequate mental model of device operation where cause and effect associations can be learnt. When faced with novel products or situations with few generic features, users typically resort to trial and error exploration of the interface [29]. By designing the product to support this type of exploration through salient feedback of its current state, users will be supported in their attempts to learn how the product works.

\section{Discussion}

In this paper, we highlighted the types of cognitive demands that could arise when interacting with consumer products. We also utilised the concept of mental models in the form of sequences of use overlaid on a state-based representation of product behaviour. This is just one possible form of representation that could enable the estimation of cognitive demands. For the purposes of evaluating cognitive product demand we do not require a model that is a precise evocation of the cognitive processes that are involved in an interaction. Nor do we require one that is capable of accounting for detailed findings and predictions of cognitive psychology. Instead, our aim is to further develop this approach into a quantitative method that can estimate various levels of cognitive demand based on simple representations of mental models of product functioning and use. This should empower designers to make decisions on how to improve the product while reducing the number of people who may be excluded or have difficulty because they do not have sufficient levels of cognitive capability to access and use the product. 


\section{Conclusions and Further Work}

A simple model of cognition was used as the basis for outlining categories of cognitive capability and demand for assessing consumer products. The concept and representation of mental models was also utilised as an approach for extracting cognitive demands. Further work involves developing quantitative measures of the outlined capabilities and investigating the predictive validity of the analytical approach to evaluation through user trials and product case studies.

Acknowledgments: This work has been funded by the UK EPSRC and the University of Trinidad and Tobago (UTT).

\section{References}

1. BSI: BS 7000-6:2005 Design management systems - Part 6: Managing inclusive design - Guide. British Standards Institute (2005)

2. Keates, S., Clarkson, J.: Countering design exclusion - An introduction to inclusive design. Springer (2003)

3. Clarkson, J., Keates, S.: A framework for minimising design exclusion. Include 2003 - Inclusive design for society and business, London (2003) 10:390 310:395

4. Clarkson, J., Keates, S.: User capabilities and product demands. Include 2003 - Inclusive design for society and business, London (2003) 10:384 - 310:388

5. Pirkl, J.J., Babic, A.L.: Guidelines and Strategies for Designing Transgenerational Products: A Resource Manual for Industrial Design Professionals. Copley Publishing Group (1988)

6. Vanderheiden, G.C., Vanderheiden, K.: Guidelines for the Design of Consumer Products to Increase Their Accessibility to People with Disabilities Working Draft 1.7. Trace R \& D Center (1992)

7. Poulson, D., Ashby, M., Richardson, S. (eds.): USERfit A practical handbook on user-centred design for assistive technology. HUSAT Research Institute, Loughborough (1996)

8. Dong, H.: Barriers to Inclusive Design in the UK. Department of Engineering. University of Cambridge, Cambridge (2004) 213

9. Goodman, J., Langdon, P.M., Clarkson, P.J.: Providing Strategic User Information for Designers: Methods and Initial Findings. In: Clarkson, J., Langdon, P., Robinson, P. (eds.): Designing Accessible Technology. Springer (2006) 41-51

10. Bridger, R.S.: Introduction to ergonomics. Taylor \& Francis (2003)

11. Hartson, H.R., Andre, T.S., Williges, R.C.: Criteria for Evaluating Usability Evaluation Methods. International Journal of Human-Computer Interaction 13 (2001) 373-410

12. Gyi, D.E., Sims, R.E., Porter, J.M., Marshall, R., Case, K.: Representing older and disabled people in virtual user trials: data collection methods. Applied Ergonomics 35 (2004) 443 - 451 
13. Persad, U., Clarkson, P.J.: Characterising Users for Inclusive Design - Three Requirements. Include 2005, Royal College of Art, London, UK (2005)

14. Carlsson, G., Iwarsson, S., Sthål, A.: The Personal Component of Accessibility at Group Level: Exploring the Complexity of Functional Capacity. Scandinavian Journal of Occupational Therapy 9 (2002) 100-108

15. Card, S.K., Moran, T.P., Newell, A.: The Psychology of Human-Computer Interaction. Lawrence Erlbaum Associates Inc (1983)

16. Grundy, E., Ahlburg, D., Ali, M., Breeze, E., Sloggett, A.: Disability in Great Britain: Results from the 1996/97 disability follow-up to the family resources survey. UK Department of Social Security (1999)

17. Langdon, P., Keates, S., Clarkson, P.: New cognitive capability scales for inclusive product design. In: Keates, S., Clarkson, J., Langdon, P., Robinson, P. (eds.): Designing a more inclusive world. Springer, London (2004)

18. Carroll, J.B.: Human cognitive abilities : a survey of factor-analytic studies. Cambridge University Press, Cambridge (1993)

19. VanDerVeer, G.C., Melguizo, M.d.C.P.: Mental Models. In: Jacko, J.A., Sears, A. (eds.): The Human-Computer Interaction Handbook: Fundamentals, Evolving Technologies and Emerging Applications. LEA, New Jersey (2003) 5280 20. Monk, A.: Cyclic interaction: a unitary approach to intention, action and the environment. Cognition 68 (1998) 95-110

21. Norman, D.A.: The Design of Everyday Things. Basic Books (Perseus), New York (2002)

22. Adams, R., Langdon, P., Clarkson, P.J.: A Systematic Basis for Developing Cognitive Assessment Methods for Assistive Technology. In: Keates, S., Langdon, P., Clarkson, P.J., Robinson, P. (eds.): Universal Access and Assistive Technology. Springer (2002) 53-62

23. Byrne, M.D.: Cognitive Architecture. In: Jacko, J.A., Sears, A. (eds.): The Human-Computer Interaction Handbook: Fundamentals, Evolving Technologies and Emerging Applications. LEA, New Jersey (2003) 97-117

24. Proctor, R.W., Vu, K.-P.L.: Human Information Processing: An Overview for Human-Computer Interaction. In: Jacko, J.A., Sears, A. (eds.): The HumanComputer Interaction Handbook: Fundamentals, Evolving Technologies and Emerging Applications. LEA, New Jersey (2003) 35-51

25. Wickens, C.D., Hollands, J.G.: Engineering Psychology and Human Performance. Prentice Hall (1999)

26. Baddeley, A.D.: The Episodic Buffer: A New Component of Working Memory? Trends in Cognitive Sciences 4 (2000) 417-423

27. Cañas, J.J., Antolí, A., Quesada, J.F.: The role of working memory on measuring mental models of physical systems. Psicologica 22 (2001) 25-42

28. Norman, D.A.: Some Observations on Mental Models. In: Gentner, D., Stevens, A.L. (eds.): Mental Models. Lawrence Erlbaum Associates (1983) 7-14.

29. Langdon, P.M., Lewis, T., Clarkson, P.J.: The Effects of Prior Experience on the Use of Consumer Products, UAIS, Special edition on Designing Accessible Techology, April 2007 (in press). 